\title{
METODOLOGÍA APLICADA A LA MEJORA \\ DE PROCESOS UTILIZANDO \\ HERRAMIENTAS DE INNOVACIÓN
}

\author{
JACQUELINE LIZARBE ${ }^{1}$ \\ https://orcid.org/0000-0002-4213-5267 \\ María Claudia Aguilar ${ }^{2}$ \\ https://orcid.org/0000-0002-8765-2647 \\ Universidad de Lima, Perú
}

Recibido: 10 de septiembre del 2019 / Aprobado: 13 de agosto del 2020

doi: https://doi.org/10.26439/ing.ind2020.n039.4920

RESUMEN: El artículo describe una técnica que utiliza herramientas de innovación para la mejora de procesos con el fin de proporcionar nuevas y mejores formas de aplicar metodologías que actualmente son utilizadas en las empresas, tales como PDCA, Six Sigma, kaizen y jidoka, entre otras. Para ello, se utilizarán herramientas que involucren a los colaboradores de las organizaciones en los procesos que no estén alcanzando los objetivos estratégicos. Esta metodología consta de seis pasos: los tres primeros están enfocados en la definición, medición y análisis del problema; los tres siguientes son el diseño, las pruebas, y la implementación y control de la solución. Esta herramienta busca que la empresa obtenga resultados disruptivos durante el proceso de análisis e implementación de mejoras, resultante de la aplicación de la metodología descrita en el presente artículo. Mediante el método del caso, se aplica la propuesta a un caso real y se validan los resultados obtenidos de la aplicación.

Palabras clave: Modelo de Procesos de Negocio (BPM) / rediseño / mejora continua / estrategia / innovación

*Correos electrónicos: 1 lizarbe@gmil.com, 2 Maguilar@ulima.edu.pe 


\section{A METHODOLOGY FOR PROCESS IMPROVEMENT USING INNOVATION TOOLS}

ABSTRACT: This article describes a technique that uses innovation tools for process improvement aimed at providing new and better ways to apply methodologies, such as PDCA, Six Sigma, kaizen and jidoka, among others, that are currently used in companies. To this end, tools that involve employees of companies whose processes are not reaching the strategic objectives will be used. This methodology consists of six steps: the first three are focused on the definition, measurement and analysis of the problem, while the next three address the design, tests, and implementation and control of the solution. This tool seeks breakthrough results for companies during the process of analysis and implementation of improvements that result from applying the methodology described herein. Using the case method, the proposed methodology is applied to a real case and its results are validated.

Keywords: Business Process Modeling (BPM) / redesign / continuous improvement / strategy / innovation 


\section{INTRODUCCIÓN}

De acuerdo con Espinoza de Los Monteros (2014), las organizaciones cada vez están más preocupadas por gestionar sus procesos y buscar nuevas formas de mejorarlos, y el Perú no es la excepción. Sin embargo, actualmente las compañías continúan utilizando herramientas y metodologías convencionales para el rediseño o mejora de procesos, tales como Six Sigma, Business Process Management (BPM) y el ciclo PDCA, entre otras. Si bien son metodologías probadas y con las cuales podemos obtener resultados que mejoren los procesos de las organizaciones, estos resultados tienen poca diferenciación frente a las mejoras realizadas en el pasado, pues no permiten la generación de mejoras disruptivas que agreguen valor a los procesos, productos y servicios debido a que no consideran un factor clave: qué realmente quiere el cliente y qué es lo que está dispuesto a pagar. El propósito de este artículo es brindar herramientas que permitan la identificación de problemas y soluciones ante los procesos de la organización que requieran ser mejorados disruptivamente, incrementando su desempeño de manera mucho más eficiente, y con estas mejoras lograr que sus clientes se sientan escuchados.

Según Ospina, el proceso consiste en "la revisión fundamental y el rediseño radical de los procesos para alcanzar mejoras espectaculares en medidas críticas y contemporáneas de rendimiento, tales como costes, calidad, servicio y rapidez" (2006, p. 34). Lo que se busca es mejorar el rendimiento de los procesos mediante la reducción de los costos o el incremento en las ventas por medio de la satisfacción de los clientes o la reducción de los tiempos de operación para la salida al mercado, entre otros aspectos que lleven la compañía a otro nivel. Sin embargo, el no conocer herramientas adecuadas que incorporen técnicas innovadoras que cuestionen lo que el cliente (tanto interno como externo) necesita, quiere y valora, y el no aplicarlas correctamente, puede llevar al fracaso de los objetivos planteados. El problema hoy en las organizaciones es cómo ser más rentables sin dejar de satisfacer las necesidades de sus clientes: "Las empresas específicamente tienen el reto de ser rentables y competitivas en este entorno caótico, donde los clientes cada vez exigen más por su dinero y tienen diversidad de opciones para cubrir sus necesidades en un mundo globalizado" (Carballo-Mendívil, ArellanoGonzales y Ríos-Vásquez, 2018, p. 63).

Entonces, ¿es posible que las organizaciones tengan resultados disruptivos en el desarrollo de proyectos de mejora de procesos haciendo uso de las herramientas adecuadas? El presente estudio se basará en la experiencia obtenida en la empresa peruana, utilizando herramientas de innovación que permiten diagnosticar problemas en los procesos de las organizaciones y proponer soluciones y mejoras disruptivas que contribuyan a alcanzar los objetivos. Para ello, se va a presentar un modelo teórico con aplicación en un caso real de la industria agroindustrial. Tal como mencionan los autores Serrano Gómez y Ortiz Pimiento (2012), “mejoramiento es adelantar, acrecentar algo, 
haciéndolo pasar a un estado mejor". También se trata de un conjunto de acciones que tiene la finalidad de aumentar la rentabilidad de la organización, mejorando aspectos tales como la calidad, el servicio, los tiempos de respuesta, los costos, etcétera.

Por otro lado, el uso de herramientas de innovación para los procesos de las organizaciones debe ofrecer respuesta a la impredecibilidad de los mercados actuales, así como a las oportunidades que surgen. El mundo de hoy ha cambiado: ahora ya no es predecible y exige cambios constantes en los procesos, servicios y productos que se ofrecen (Robayo Acuña, 2016). El método propuesto tiene las siguientes fases: diagnóstico de los procesos, identificación de posibles oportunidades de mejora, búsqueda de problemas y oportunidades de mejora, ideación de las mejoras, y finalmente, prototipado o prueba de las soluciones propuestas.

Para el diagnóstico de los procesos, dentro de una organización existen algunas técnicas de recolección de información. Uno de los objetivos es mostrar elementos que resulten útiles para las diferentes etapas en la mejora de procesos; por ejemplo, durante las reuniones iniciales de relevamiento de procesos con el personal involucrado en el desarrollo de las actividades, se requiere utilizar una herramienta práctica, ágil y completa que facilite la explicación del proceso de inicio a fin, considerando que se debe mencionar las actividades, los responsables, los recursos necesarios en cada proceso (información, tecnología, personal involucrado maquinaria, etcétera), y la frecuencia. Existen buenas herramientas y gráficas que cumplen con las características mencionadas, tales como el diagrama SIPOC, el diagrama de flujo o el diagrama de recorrido, para mostrar gráficamente el proceso que se está evaluando.

El diagrama SIPOC, por sus siglas en inglés, significa Suppliers, Input, Process (Actividades), Output y Customer. Según Gueorguiev (2018), describe a un nivel muy profundo el desarrollo de un proceso, identificando a las personas dentro y fuera de la organización que proveen las entradas en cada parte del proceso, los recursos utilizados, el paso a paso de las actividades que se realizan, los resultados producto de la transformación de cada uno de los recursos que ingresaron al sistema, y finalmente, el cliente interno o externo, aquel que recibe los productos o servicios resultantes de cada parte del proceso. Por ello es uno de los diagramas más usados por las consultoras que trabajan en mejora de procesos. Otro objetivo de la investigación es proporcionar las herramientas adecuadas para que el equipo encargado de la mejora pueda proponer soluciones que hagan procesos más eficientes, basadas en reducir tiempo en las actividades, reducir costos y mejorar la calidad de los productos o servicios, lo que se traduce en un incremento de la productividad.

El flujo que describe el proceso actual ( $A s / s$ ) y el futuro ( $T o B e$ ) muestra el trabajo realizado para la identificación de mejoras, las cuales son evaluadas con la finalidad de medir el impacto generado sobre el costo que implica realizar los cambios. Estos 
flujos pueden ser diagramados utilizando diversas metodologías, tales como diagrama de bloques, diagrama de Operaciones de Procesos (DOP), diagrama de Business Process Management (BPM) o un diagrama de Actividades de Proceso (DAP). Estos diagramas muestran de inicio a fin cómo funcionan los procesos en la organización. El diagrama de bloques, el DOP y el BPM incluyen principalmente actividades de operación, actividades de control y toma de decisiones. Uno de los softwares más utilizados para el modelamiento de procesos es Bizagi, sistema que utiliza la metodología BPM para diagramar procesos.

Con el objetivo de mejorar los procesos que se evalúan en las organizaciones y llevar un flujo As ls a una versión To Be, se requiere identificar los puntos de atención o problemas detectados en los procesos actuales para ser analizados con herramientas estadísticas sobre datos históricos que contengan volumen, frecuencia de las transacciones, periodicidad y costos o ingresos, según el tipo de información que es preciso analizar. Esta información se debe comparar con el resultado del mismo análisis realizado a la propuesta de mejora, con la finalidad de decidir si es la solución correcta para el problema y la organización debe invertir en ella.

Para implementar una propuesta de mejora en el proceso y con las personas que son parte de él, es necesario probarla y verificarla entre dos a cuatro meses, dependiendo de la naturaleza del cambio; si no hay resultados, se consideran opciones que funcionen mejor o, de ser necesario, se desarrollará nuevamente todo el proceso de mejora desde el diagnóstico hasta la implementación de la nueva solución. Los consultores en mejora o rediseño de procesos creen conveniente esperar dos meses con la mejora en funcionamiento en condiciones estándar para comprobar su adecuada implementación.

Las herramientas que se busca proponer en la presente investigación están orientadas a la mejora de procesos de forma radical en empresas de manufactura o de servicios que tengan procesos que agreguen valor al consumidor final, tales como procesos de producción, de atención al cliente, de ejecución de un servicio, de comercialización y ventas, entre otros. Según Harrington (1992), agregar valor es aquello que el cliente pagaría con gusto y que brinda una ventaja competitiva a la organización.

\section{MATERIALES Y MÉTODOS}

A continuación, se presenta una herramienta propuesta para realizar proyectos de mejora de procesos en organizaciones industriales y de servicios que busquen obtener soluciones disruptivas e incrementales. La presente herramienta consta de seis pasos para la mejora de procesos, que incluyen metodologías de innovación para la obtención de resultados disruptivos. 
Primer paso. Identificación del o los procesos a innovar

Se realiza un taller de identificación de los objetivos que se pretende conseguir. Dentro de este taller se deben determinar aquellos que no están siendo alcanzados por problemas en los procesos. Los procesos a mejorar que se identifiquen deben ser priorizados en función de los objetivos estratégicos de la organización, y con ello empieza el trabajo de innovación. El taller se realiza con el modelo Canvas, que permite tener una idea clara del negocio, sus procesos clave y a dónde se quiere llegar. Los colaboradores que forman parte del taller deben tener conocimiento de la estrategia y la visión del negocio. Los resultados del taller deben identificar los procesos en los que hay que trabajar para encontrar una solución o generar una mejora que impacte en el beneficio de la organización, sus clientes y sus colaboradores.

Segundo paso. Identificación de oportunidades de mejora - usuarios del proceso

Conformar equipos de doce a quince colaboradores de diferentes áreas de la organización, aun cuando no tengan ninguna relación con el proceso seleccionado. En la primera sesión del equipo, se aplicará la metodología del Brown Paper, utilizada en consultoras del mundo tales como Synergos y BDO. Esta herramienta servirá para describir los procesos seleccionados y que todo el equipo tenga una clara comprensión de las actividades que se realizan, cómo se realizan y dónde está el "dolor" en el proceso, es decir, que se puedan identificar aquellas oportunidades de mejora que deberán ser los puntos clave en donde se buscará la innovación. Como indica Harrington (1992), se debe obtener el conocimiento del proceso a partir de las actividades que la conforman, elaborando un diagrama de flujo preliminar que permita esquematizar el proceso para su fácil comprensión.

Tercer paso. Definición del problema - cliente (interno y externo)

En esta etapa se aplica el modelo de empatía explicado en el texto Generación de modelos de negocio (Osterwalder y Pigneur, 2011). Para lograr el entendimiento de los problemas y "dolores" dentro del proceso evaluado, debemos preguntar "por qué" tantas veces como sea necesario, hasta encontrar los problemas reales en una reunión de empatía con los clientes. Al finalizar, la información recopilada de los clientes debe ser llevada a un taller para definir el problema, utilizando el mapa de empatía en donde se realizan preguntas tales como:

1. ¿Qué piensa y siente el observado?

2. ¿Qué oye el observado?

3. ¿Qué ve el observado?

4. ¿Qué dice y hace el observado en la reunión? 
5. ¿Cuáles son los miedos, frustraciones y obstáculos que tiene nuestro observado?

6. ¿Cuáles son los deseos, necesidades y medidas de éxito de nuestro observado?

La metodología ISO 9004 y Harrington proponen que es indispensable la identificación de las causas de los problemas en los procesos con la finalidad de tener un diagnóstico; por ello, las entrevistas a los clientes internos y externos involucrados en el proceso se convierten en la fuente principal para obtener las causas raíces de los problemas que se detecten.

Cuarto paso. Ideando la solución

En un taller de empatía se debe aplicar la metodología de Scamper, que consiste en definir una lista de siete preguntas con el objetivo de estimular la generación de ideas con respecto al proceso seleccionado (Tarapuez y Lima, 2013).

1. ¿Qué podemos sustituir dentro del proceso para solucionar los problemas o dolores encontrados en la etapa de empatía?

2. ¿Qué actividades o recursos podemos combinar para obtener un mejor resultado?

3. ¿Es posible adaptar algo a una parte del proceso?

4. ¿Es posible modificar una actividad?

5. ¿Es posible dar otro uso a un recurso o activo dentro del proceso?

6. ¿Es factible eliminar una actividad, función o recurso?

7. ¿Es factible reordenar o invertir las actividades del proceso?

Dentro del mismo taller se puede también utilizar la técnica de evaluación de cuatro frentes: paradigmas, necesidades del cliente o usuario, tendencias y recursos del interior de la empresa o de terceros. Los paradigmas buscarán encontrar aquellas situaciones o reglas que se asumen como la única forma de realizar las actividades que debemos validar, modificar o eliminar.

Gracias al taller de empatía, se toman las necesidades de los clientes o usuarios con la finalidad de resolverlas e idear oportunidades de mejora. Cada idea propuesta debe ser descrita considerando dar respuesta a las siguientes preguntas: ¿Qué es? ¿Qué necesidad cubre? ¿Para quién es? ¿Quién debe realizarlo? ¿Cómo resolverá el problema?

Según el libro Generación de modelos de negocio, de Osterwalder y Pigneur (2011), el proceso de ideación consiste en desafiar las normas para diseñar modelos originales que satisfagan las necesidades desatendidas, nuevas u ocultas de los clientes. En esta etapa surge diversidad de ideas, las cuales finalmente convergen en una propuesta de solución adecuada para los problemas detectados. 
Quinto paso. Prototipar la propuesta de solución

Este paso comienza con la creación de un prototipo, para luego realizar pruebas con usuarios o clientes externos del proceso. Si se trata de un cambio de sistema, se puede plantear un esquema manual que muestre cómo funciona. Si se trata de un servicio, se pueden esquematizar los cambios en el servicio de forma gráfica para explicar su funcionamiento. Luego se invita a los clientes, como participantes, para que estos interactúen con los prototipos sin que los responsables de la solución intervengan. Luego se preguntará si se entienden los cambios propuestos, si es lo que realmente estaban buscando y si satisfacen sus expectativas o se deben hacer modificaciones. Si existen modificaciones, estas se realizan y se vuelven a presentar hasta que se llegue a la conformidad de los usuarios. Como mencionan Osterwalder y Pigneur (2011), no se trata de desarrollar ideas que se pretenda realizar sino de explorar ideas nuevas mediante la adición o eliminación de elementos al prototipo que se está testeando ante los clientes internos y externos.

Sexto paso. Implementación de la solución

Si el prototipo ya probado se ajusta a lo esperado y responde a los problemas de los clientes, entonces es el momento de implementar la solución creada. La implementación requiere la modificación de los documentos actuales, tales como procedimientos, funciones, políticas, protocolos, manuales e instructivos, entre otros, y la capacitación de los usuarios involucrados en las modificaciones realizadas. Asimismo, para asegurar el cambio se deberá monitorear que los nuevos procesos estén funcionando en el periodo de dos a cuatro meses que puede durar la estabilización de las nuevas actividades o que permita ajustar el desempeño del proceso con las mejoras aplicadas. Todos los cambios propuestos y aprobados deben estar documentados, de tal forma que puedan ser conocidos por todos los involucrados para que los nuevos usuarios puedan acceder al material y así comprender el funcionamiento del proceso mejorado. Tal como indican Pérez Ortega y Soto Camargo (2005) sobre el seguimiento a las mejoras realizadas, se deben medir mediante indicadores de eficiencia, efectividad y encuestas de satisfacción, entre otros.

El programa de capacitación al personal involucrado debe incluir dinámicas y talleres para que pueda ser fácilmente aprendido y adoptado por todos. En general, todas las metodologías enfocadas en la mejora continua siguen una misma línea para la realización de la mejora de los procesos. Por ejemplo, la metodología de análisis y solución de problemas (MASP) es un proceso que consta de ocho etapas que consiste en la identificación de los problemas y la propuesta de mejoras preventivas y correctivas; sin embargo, la metodología presentada en este artículo pretende ir más allá y se enfoca en descubrir las necesidades insatisfechas de los clientes internos y externos para que la propuesta de solución consiga superar las expectativas del cliente. En la tabla 1 se 


\section{muestran las diferencias entre la metodología propuesta y la metodología clásica PDCA de la mejora continua.}

Tabla 1

Diferencia entre la metodología propuesta y la metodología clásica PDCA (mejora continua)

Metodología propuesta

Consta de cuatro etapas marcadas: diagnóstico de la situación actual, análisis de la situación actual, diseño de la o las posibles soluciones e implementación de las soluciones aprobadas.

El diagnóstico incluye el trazado de objetivos que se deben cumplir en el rediseño de los procesos de la organización y su alineación con la estrategia del negocio.

La búsqueda de oportunidades de mejora y la detección de problemas se preocupa por involucrar a colaboradores, involucrados o no en el proceso, que puedan tener diferentes perspectivas sobre él y sobre todo que tengan las habilidades de creatividad necesarias para participar en el proceso.

La metodología propuesta involucra también a los clientes externos para la detección de necesidades y requerimientos no cubiertas y la problemática que pudieran presentar.

Utiliza herramientas específicas para la detección de las necesidades, requerimientos y problemas de los clientes internos y externos.

El diseño de la solución involucra el desarrollo de prototipos que testeen con el cliente interno y externo el cumplimiento de sus necesidades y requerimientos, así como la solución a sus problemas.

Los prototipos realizados son a pequeña escala, incluso a manera de maqueta en primera instancia, para evitar gastos innecesarios antes de las validaciones con los clientes. A partir del primer prototipo realizado se va construyendo la solución final.
Metodología PDCA

Consta de cuatro etapas: planificar los objetivos del trabajo de mejora, hacer lo que se ha planeado, verificar lo que se ha realizado y la etapa de actuar, donde se corrigen errores.

Se definen objetivos para la mejora, pero no están necesariamente relacionados con la estrategia del negocio.

La detección del problema y de las oportunidades de mejora involucra solo al personal que está directamente relacionado con la ejecución de los procesos, sin considerar colaboradores externos que puedan aportar.

No involucra al cliente externo en el proceso de mejora.

No se utilizan herramientas específicas para captar las necesidades, requerimientos y problemática de los clientes.

No se realizan prototipos.

Al no haber prototipos luego de la implementación a pequeña o gran escala, se verifican los resultados y se corrige. Las correcciones que resulten pueden ser muy costosas.

Elaboración propia

\section{RESULTADOS}

Para la realización de la mejora de procesos se utilizaron datos proporcionados por la organización, tales como objetivos de la empresa, metas, indicadores y resultados 
obtenidos, mapa de procesos, datos de producción y de ventas relevantes para la identificación de las oportunidades de mejora y análisis de las propuestas de solución. A continuación, se presenta una aplicación práctica de los pasos de la metodología.

CASO: Empresa agroindustrial

PROCESO: Proceso de siembra y cosecha de arándanos

Paso 1. Identificación del o los procesos a innovar

El punto inicial es comprender el modelo de negocio de la empresa y sus lineamientos estratégicos, y a su vez, identificar aquellos objetivos que no se está logrando cumplir a causa de procesos obsoletos o mal definidos por los líderes del negocio. En la tabla 2 se muestran los objetivos de la organización evaluada conforme a su plan estratégico.

Tabla 2

Objetivos estratégicos de la empresa

\begin{tabular}{|c|c|}
\hline Frentes & Objetivos \\
\hline Financiero & $\begin{array}{l}\text { Lograr una rentabilidad del } 13 \% \text { en la colocación de arándanos en el mercado } \\
\text { norteamericano. }\end{array}$ \\
\hline Procesos & Aumentar la producción de arándanos de primera calidad en un 9 \%. \\
\hline Cliente & Incrementar la fidelidad de nuestros clientes del exterior del país. \\
\hline Aprendizaje & $\begin{array}{l}\text { Capacitar en nuevas técnicas de siembra y cuidado de los cultivos de aránda- } \\
\text { nos a nuestros operarios de campo. }\end{array}$ \\
\hline
\end{tabular}

Elaboración propia

Los objetivos estratégicos de la organización deben ser validados con los resultados obtenidos en los últimos dos años para identificar cuáles requieren ser revisados y mejorados. En la tabla 3 se muestra el modelo Canvas de la organización, donde en un solo esquema se puede observar la razón de ser de la empresa, su propuesta de valor y la forma en que puede hacer entrega de esta a sus clientes. 
Tabla 3

Modelo de negocio Canvas de la agroindustria

\begin{tabular}{|c|c|c|c|c|}
\hline $\begin{array}{l}\text { Socios } \\
\text { clave }\end{array}$ & $\begin{array}{l}\text { Actividades } \\
\text { clave }\end{array}$ & Propuesta de valor & Relación con el cliente & Segmento de cliente \\
\hline \multirow{3}{*}{$\begin{array}{l}\text { Naviera } \\
\text { Operarios }\end{array}$} & $\begin{array}{l}\text { Logística } \\
\text { Siembra } \\
\text { Cosecha } \\
\text { Reclutamiento }\end{array}$ & \multirow{3}{*}{$\begin{array}{l}\text { Producto con alta } \\
\text { calidad de la fruta } \\
\text { Cumplimiento de } \\
\text { estándares } \\
\text { Certificación ISO, } \\
\text { HACCP y Comercio } \\
\text { Seguro }\end{array}$} & $\begin{array}{l}\text { Redes sociales } \\
\text { corporativas } \\
\text { Correo corporativo } \\
\text { Servicio de tracking de } \\
\text { pedidos }\end{array}$ & \multirow{3}{*}{$\begin{array}{l}\text { EE. UU. } \\
\text { Asia } \\
\text { Europa } \\
\text { Nacional }\end{array}$} \\
\hline & Recursos clave & & Canales & \\
\hline & $\begin{array}{l}\text { Colaboradores } \\
\text { Maquinaria } \\
\text { Insumos de } \\
\text { cultivo }\end{array}$ & & $\begin{array}{l}\text { Vía aérea } \\
\text { Vía marítima }\end{array}$ & \\
\hline \multicolumn{2}{|c|}{ Costos clave } & & \multicolumn{2}{|l|}{ Ingresos clave } \\
\hline $\begin{array}{l}\text { Horas máqu } \\
\text { Horas homb } \\
\text { Transporte }\end{array}$ & & & \multicolumn{2}{|l|}{$\begin{array}{l}\text { Venta de fruta en el país } \\
\text { Venta de fruta al exterior }\end{array}$} \\
\hline
\end{tabular}

Elaboración propia

Para la realización del Canvas se proporcionan valores como: proporción por tipo de segmento de cliente, nivel de satisfacción por cada segmento de cliente, valor en venta por producto, valor de los costos y gastos más importantes de la empresa.

Conforme a los resultados proporcionados por la organización, donde la producción de arándanos de primera calidad solo ha aumentado en un $5 \%$ con respecto al año anterior, se infiere claramente que el objetivo que la empresa no ha alcanzado es el de procesos, ya que no ha logrado aumentar la producción de arándanos de primera calidad en un $9 \%$. Por ello, se seleccionó como proceso a mejorar la siembra y cosecha de arándanos.

Paso 2. Identificación de oportunidades de mejora - usuarios del proceso

En esta etapa se realizó un taller seleccionando el equipo de innovación ideal para realizar el flujo de procesos utilizando la herramienta Brown Paper, diagramando la forma actual como se realizan los procesos de siembra y de cosecha de los arándanos.

En la figura 1 se muestra el flujo de siembra y cosecha de arándanos con la identificación de oportunidades de mejora. 


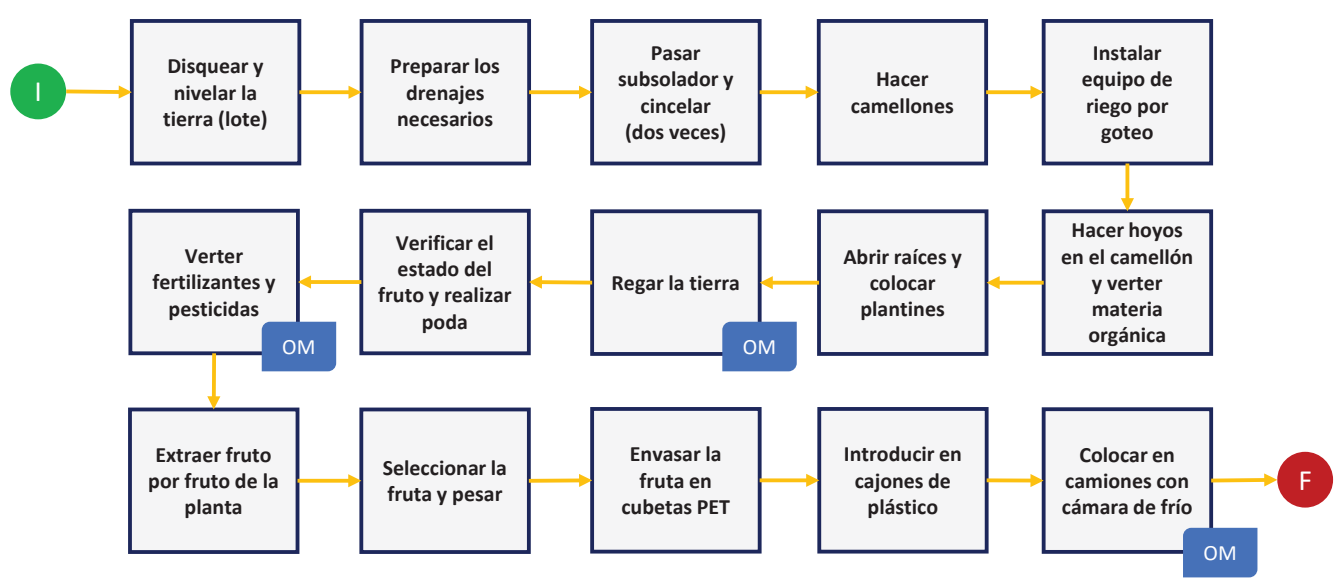

Figura 1. Proceso de siembra y cosecha de arándanos

Elaboración propia

En el flujo se han identificado las oportunidades de mejora (OM) detectadas con postits de color celeste.

Las oportunidades de mejora más importantes encontradas son:

a. No es posible predecir con certeza el consumo de fertilizantes y pesticidas en las parcelas al no contar con información histórica de la utilización de estos recursos y de las enfermedades de esta planta durante su cultivo.

b. Es difícil conocer el consumo de metros cúbicos de agua para una parcela de arándanos, ya que no se cuenta con información histórica confiable y real para pronosticar la aplicación de este recurso en forma adecuada.

c. Se tiene dificultad en coordinar la logística adecuada para la cosecha, pues no es fácil de pronosticar la cantidad de arándano que resultará de los campos.

Paso 3. Definición del problema - clientes (interno y externo)

En este paso se debe primero identificar quién es el cliente principal del flujo de siembra y cosecha de arándanos y el objetivo que se está por alcanzar: aumentar en 9 \% la producción de arándanos de primera calidad. Los clientes más importantes de este flujo son:

a. Los cosechadores, quienes retiran todos los frutos que han sido cultivados y sembrados.

b. Los clientes finales, quienes adquieren los arándanos de la empresa. 
Con estos clientes internos y externos se realiza el taller de empatía, y este deberá ser por separado. No es necesario contestar todas las preguntas, pero sí debemos entender qué siente el observado, cuáles son sus preocupaciones y qué es lo que le gustaría mejorar o cambiar.

Para que sea exitoso, el taller debe utilizar el formato que se muestra en la figura 2, lo que permitirá que todos los participantes del taller, tanto los encargados como los clientes, estén alineados con las preguntas que se deben realizar y con las respuestas que se desea obtener.

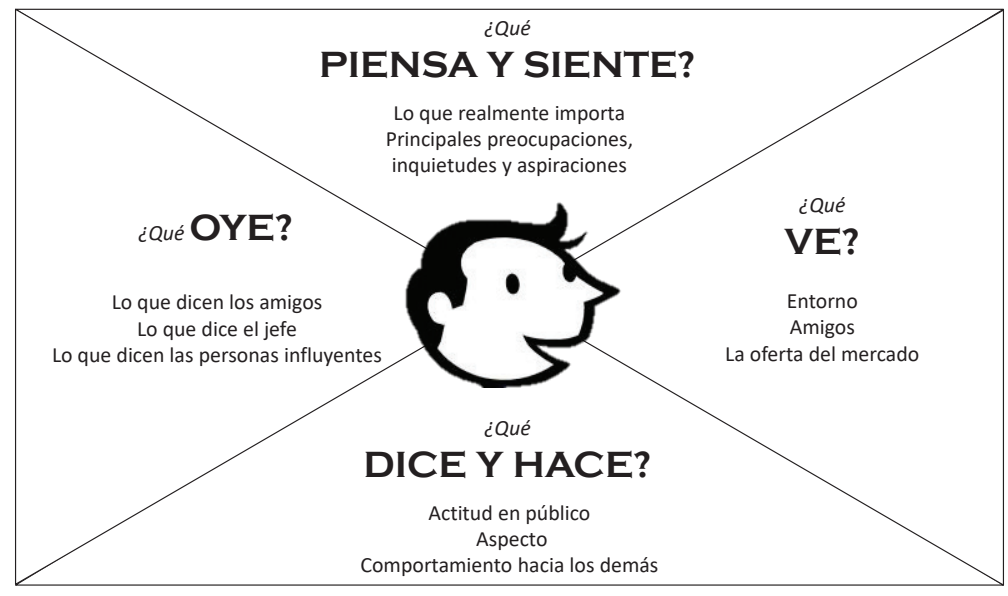

\begin{tabular}{|c|c|}
\hline ESFUERZOS & RESULTADOS \\
Miedos \\
$\begin{array}{c}\text { Frustraciones } \\
\text { Obstáculos }\end{array}$ & $\begin{array}{c}\text { Deseos/necesidades } \\
\text { Medidas de éxito } \\
\text { Obstáculos }\end{array}$ \\
\hline
\end{tabular}

Figura 2. Mapa de empatía

Fuente: Osterwalder y Pigneur (2011)

\section{Clientes internos/cosechadores}

1. ¿Qué piensan y sienten con respecto al proceso de siembra y cosecha de arándanos?

Objetivo. Detallar lo que les importa realmente a los cosechadores con respecto al proceso interno o servicio que se les ofrece: sus preocupaciones, inquietudes y aspiraciones.

- Los cosechadores estarían gustosos de conocer la cantidad precisa de fertilizantes y pesticidas a utilizar en los campos, de tal manera de conservar la mejor calidad posible de los arándanos hasta la cosecha. 
- Muestran su preocupación por la calidad del arándano que deben retirar de los campos, ya que de eso depende en gran parte la calidad del producto final.

- Están preocupados por tener una herramienta que agilice el proceso de registro de las cantidades cosechadas de arándano de las distintas calidades.

2. ¿Qué oyen respecto del proceso de siembra y cosecha de arándanos?

Objetivo. Reconocer quiénes son las personas que tienen poder en la decisión y el actuar de los cosechadores, y qué dicen acerca de los procesos internos o del servicio que se les ofrece. Se debe saber qué escuchan los cosechadores de sus amigos, compañeros de trabajo u otros usuarios del proceso.

- El área de calidad de cultivos menciona que, al no tener datos históricos de las enfermedades que ocurren durante el tiempo de cultivo y las cantidades de fertilizantes y pesticidas utilizados para contrarrestar esta situación, no es posible predecir la cantidad más adecuada a verter en los campos para lograr obtener una mejora en la calidad del producto final.

3. ¿Cuáles son los miedos, frustraciones y obstáculos que tienen respecto del proceso de siembra y cosecha de arándanos?

Objetivo. Entender qué limita a los cosechadores de poder interactuar con el proceso o el servicio que se les ofrece, y saber qué obstáculos deben enfrentar para tener un buen resultado en el proceso evaluado o recibir un buen producto.

- Mantener y cuidar la calidad de los cultivos es lo más importante, pero no es sencillo, ya que no se cuenta con información histórica y pronósticos de cultivos anteriores que ayuden a predecir cómo actuar en el presente. Hasta cierto punto es frustrante trabajar a partir de supuestos, mas no de información real.

- Conforme pasan los días de cultivo y los días de cosecha van llegando, todas las herramientas de cosecha deben estar preparadas con anticipación para no tener ningún inconveniente, ya que la calidad del producto disminuye con el paso del tiempo.

4. ¿Cuáles son los deseos, necesidades y medidas de éxito con respecto al proceso de siembra y cosecha de arándanos?

Objetivo. Descubrir qué desean o necesitan los cosechadores para que el proceso evaluado mejore o para que el producto brindado cumpla sus expectativas. Se debe saber cuáles son las medidas que indican el éxito del proceso o del producto evaluado. 
- Lo ideal es contar con información histórica de los cultivos y registrar de forma inmediata todas las variables de la siembra, cultivo y cosecha, tales como metros cúbicos de agua, litros de fertilizante, litros de pesticidas, tipos de fertilizantes y pesticidas utilizados y plagas ocurridas, entre otros. Esto permitirá invertir mejor en fertilizantes, pesticidas, etcétera.

- Además, se deberá contar con pronósticos más acertados que indiquen cuánta cantidad de arándano se cosechará, y con ello, poder definir la logística necesaria para el transporte y despacho.

\section{Clientes externos / compradores}

1. ¿Qué piensa y siente el observado con respecto al proceso de siembra y cosecha de arándanos?

Objetivo. Detallar lo que les importa realmente a los clientes o compradores con respecto al proceso interno o servicio al cliente que se les ofrece: sus preocupaciones, inquietudes y aspiraciones.

- Los clientes tienen cada vez más exigencias en cuanto a la calidad del arándano que quieren consumir. Entre las características pedidas están: producto fresco, dimensiones estándar y de buen color.

- En cuanto a la presentación, los clientes buscan cada vez más que la forma en la que se envasa el producto sea llamativa a la vista para un público internacional exigente.

- Los clientes están preocupados por adquirir arándanos con menor riesgo de daño en el consumo humano debido a pesticidas o fertilizantes utilizados en los cultivos.

2. ¿Qué oyen con respecto al proceso de siembra y cosecha de arándanos?

Objetivo. Reconocer quiénes son las personas que tienen poder en la decisión y el actuar de los clientes/compradores, y qué dicen acerca de los procesos internos o del producto que se les ofrece. Se debe saber qué escuchan los clientes o compradores sobre sus colaboradores, proveedores, competencia u otros usuarios del producto.

- El cliente está en constante comparación en cuanto a la compra de arándano en el mundo y cuenta con información disponible sobre la producción de estos cultivos en otros países, como Chile, México y Canadá.

- Se debe saber que el cliente puede comparar precios, calidades y presentación de los arándanos de cada país y empresa y está dispuesto a pagar un poco más por adquirir el mejor producto que cumpla con sus exigencias. 
3. ¿Cuáles son los miedos, frustraciones y obstáculos que tiene el observado?

Objetivo. Entender qué limita a los clientes o compradores a poder interactuar con el proceso o el producto que se les ofrece, y conocer qué obstáculos se deben enfrentar para tener un buen resultado en el proceso evaluado o recibir un buen producto.

- La calidad del producto final es lo que el cliente considera más importante. La calidad no implica solo cómo se ve el fruto por fuera en cuanto a la presentación ofrecida, que de por sí ya es importante, sino sobre todo asegurar que el producto tenga las características y condiciones de salubridad requeridas para no afectar a los consumidores de mercados tan exigentes como los de Estados Unidos y Europa.

4. ¿Cuáles son los deseos, necesidades y medidas de éxito del observado?

Objetivo. Descubrir qué desean o necesitan los clientes o compradores que se cumpla, que el proceso evaluado mejore y el producto cumpla las expectativas. Se debe conocer cuáles son las medidas que indican el éxito del proceso o del producto evaluado.

- La empresa debe cubrir las necesidades esenciales de sus clientes: cumplir con la calidad del fruto, bajo contenido de fertilizantes y pesticidas, mantener las características organolépticas requeridas para el fruto (color, textura, sabor, olor) y cumplir con los tiempos de entrega.

Con todo ello, podemos indicar que el problema a resolver es mantener y mejorar la calidad del fruto para públicos tan exigentes como el de Estados Unidos y Europa, que son los principales compradores del arándano. Estas características son: organolépticas (color, textura, volumen, sabor y olor) y características de calidad del futo para el consumo humano (menos pesticidas y fertilizantes sintéticos en el fruto).

Paso 4. Ideando la solución

En esta etapa se realizó el taller de cuatro frentes: paradigmas, recursos, tendencias y necesidades. A continuación, se presentan algunas de las ideas que surgieron en este taller:

1. Paradigmas:

- No es posible tener información histórica de la siembra, cultivo y cosecha.

- No es posible conocer cuántos litros de fertilizantes y pesticidas debemos verter exactamente en los campos.

- No es posible prevenir una plaga en los cultivos.

- No es posible disminuir el margen de error en la cosecha. 
- No es posible mejorar la calidad de los cultivos.

2. Recursos:

- Personal de sistemas capacitado.

- Personal en investigación y desarrollo especializado en pronóstico y cultivos.

- Herramientas de automatización de cultivos en el mercado.

- Capacidad de inversión.

3. Tendencias:

- Aplicaciones de registro automático desde los campos de cultivo.

- Herramientas de software que ayudan con pronósticos.

4. Necesidades:

Mejorar la calidad de los productos que resultan de la cosecha.

Preparar con tiempo la logística necesaria para cada cosecha.

Prevenir las enfermedades en los campos para disminuir el uso de pesticidas.

Como producto final del taller, se debe crear la idea o las ideas de mejora al proceso que atienda las necesidades de los campos de cultivo y que mejore la calidad del producto final que es la mayor preocupación de la organización. Para la creación de las ideas de mejora se utiliza la estructura aprendida en esta etapa:

1. ¿Qué es?

- Es una aplicación que permite ingresar directamente del campo la cantidad de metros cúbicos de agua, fertilizantes y pesticidas vertidos en los campos.

- Es un software que permite predecir la cantidad de arándanos a cosechar por cada cultivo.

- Es un software que permite determinar si los cultivos tienen riesgo de contraer una enfermedad durante la etapa de cultivo.

2. ¿Qué necesidad cubre?

- Permite agilizar el proceso de registro de cada elemento que se vierte en los campos y colabora con la obtención de data histórica registrada en el sistema.

- Se puede obtener un aproximado cercano de la cantidad de arándanos a cosechar por cada cultivo para poder coordinar mejor la logística necesaria. 
- Es posible predecir si los campos obtendrán algún tipo de enfermedad que afecte a los cultivos, y con ello poder tomar con tiempo las decisiones respectivas.

3. ¿Para quién es?

- Para el personal de cultivo.

- Para el personal de cosecha y logística.

- Para el personal de sanidad y cultivo.

4. ¿Quién debe realizarlo?

- El personal de sistemas en conjunto con el personal de campo.

- El personal de sistemas en conjunto con el personal de sanidad.

5. ¿Cómo resolverá el problema?

- Ayudará a tener la información registrada a tiempo, permitiendo contar con datos históricos y reales ingresada por el personal de campo de forma rápida y automática.

- Permitirá estar preparados con la logística para la cosecha.

- Permitirá predecir las enfermedades en los campos y estar preparados antes de que ocurran y mejorar así la calidad de los cultivos.

Paso 5. Prototipar la propuesta de solución

El equipo generador de ideas en esta etapa realiza un prototipo físico del software requerido que cumple con las funcionalidades mencionadas. El prototipo está listo para ser aprobado por todos los participantes del proceso, principalmente los clientes. En esta etapa el objetivo es la iteración. Si algo no funciona y debe ser modificado se modifica el prototipo y se vuelve a probar hasta obtener el prototipo final.

Paso 6. Implementación de la solución

Para poner en práctica este paso es necesario desarrollar el prototipo, probarlo, hacer las modificaciones correspondientes, ponerlo en marcha y monitorear uno o dos meses el funcionamiento del software desarrollado, así como proponer indicadores de medición que permitan evaluar el desempeño del proceso con el nuevo software implementado.

\section{DISCUSIÓN}

La metodología propuesta ha sido aplicada en los sectores de agroindustria, pesca y comercio minorista en un total de seis empresas, de las cuales se utilizó el trabajo realizado en una de ellas como caso para la presente investigación, obteniendo resultados 
exitosos en cuanto a la mejora y rediseño de sus procesos y logrando el involucramiento de los colaboradores y clientes de la organización.

Las conclusiones más relevantes de la presente investigación son:

- La metodología y herramientas utilizadas por las empresas en la actualidad para la mejora de procesos se han visto modificadas por medio de herramientas de innovación que permiten el aporte y participación de los colaboradores y clientes de la empresa. Como indican Marin, Pardo del Val y Bonavia (2008), los grupos participantes deben pertenecer a diferentes áreas de la empresa, lo que permite compartir conocimientos y puntos de vista complementarios, así como también comprender el trabajo de otras secciones y aprender de las experiencias de los demás.

- Para iniciar una mejora de procesos, se debe analizar la estrategia del negocio y los objetivos que persiguen. Es muy importante conocer las metas planteadas de la organización y priorizar aquellas en la que no se está cumpliendo para detectar los procesos que no están contribuyendo a que se logre la meta.

- Con la finalidad de comprender el proceso que debe ser mejorado, la metodología incluye, como parte de la etapa de definición del problema, la realización de un diagrama de procesos que explique al detalle las actividades que permitan identificar las oportunidades de mejora que afectan a los clientes y colaboradores, y al logro del objetivo de la organización que no haya sido alcanzado.

- La diferencia principal entre una mejora de procesos solo usando herramientas disponibles por la empresa y una mejora de procesos con metodología de herramientas de innovación es que esta última está debidamente estructurada y considera las necesidades, frustraciones y preocupaciones de los clientes, ya sean internos o externos, que interactúan con el proceso que se va a mejorar.

- La tarea de identificar al o los clientes externos del proceso a mejorar tiene un significativo grado de dificultad, debido a que se debe considerar como tal a aquellos participantes que interactúan en el proceso, tales como receptores del resultado de la realización de las actividades del proceso.

- Los talleres de empatía como parte de la etapa de análisis del problema se realizan con los clientes del proceso buscando conocer cómo piensan, cómo se sienten durante el desarrollo del proceso, qué escuchan o ven de sus compañeros, quiénes tienen influencia en ellos y, finalmente, cuáles son sus frustraciones, necesidades y requerimientos.

- Todo lo que se pueda recopilar de los clientes contribuirá al desarrollo de las mejoras en los procesos evaluados y permitirá incrementar la satisfacción de los consumidores finales y clientes internos, según sea el caso. 
- Para la generación de las propuestas de mejora como parte de la etapa de ideación o diseño de la solución, se debe lograr mantener abierta la mente del equipo de trabajo, utilizando herramientas rompehielos para que se desenvuelvan en el taller sin problema.

- El proceso de generación de ideas es como un embudo. Al inicio surgirá gran cantidad de ideas; ninguna debe ser desestimada. A lo largo de la discusión, y con herramientas como Scamper y 4 frentes, se deberán ir aterrizando las ideas de mejora que se van obteniendo, para finalmente establecer aquellas que cobren mayor relevancia. Finalizado el proceso de generación de ideas, se inicia la etapa de pruebas, que implica realizar prototipos rápidos sobre las ideas que resultaron, las cuales deben ser aprobadas por los clientes del proceso identificado. En este proceso de iteración se debe captar todo aquello que implique mejorar o modificar el prototipo antes de poner en marcha la implementación real del o de las ideas que se están probando.

- Finalmente, todo proyecto de implementación debe culminar con una fase de seguimiento y control que implica el acompañamiento de aquellos que aplicarán la nueva forma de trabajo dentro del proceso y se podrá evaluar el desempeño de las mejoras con los clientes identificados durante la ejecución de la metodología.

\section{REFERENCIAS}

Carballo-Mendívil, B.; Arellano-Gonzáles, A. y Ríos-Vásquez N. J. (2018). La gestión de procesos esbeltos como principio de mejora. Un caso aplicado a una comercializadora. 3C empresa: investigación y pensamiento crítico, 7(3), 60-81. doi: http:// dx.doi.org/10.177993/3cemp.2018.070335.60-81/

Espinoza de Los Monteros, A. (2014). REATA: Reingeniería Estratégica de Alta Tecnología Aplicada. México: Lagares de México.

Gueorguiev, T. (2018). Improving the Internal Auditing Procedure by Using SIPOC Diagrams. Journal of Innovations and Sustainability, 4(2), 35-43.

Harrington, H. (1992). Mejoramiento de los procesos de la empresa. Colombia: McGraw-Hill.

Marin, J.; Pardo del Val, M. y Bonavia, T. (2008). La mejora continua como innovación incremental: el caso de una empresa industrial española. Economía Industrial, $368,155-167$.

Ospina, 0. (2006). Reingeniería de procesos de negocio. Colombia: Cuadernos Latinoamericanos de Administración. 
Osterwalder, A. y Pigneur, Y. (2011). Generación de modelos de negocio. España: Deusto.

Pérez Ortega, G. y Soto Camargo, A. M. (2005). Propuesta metodológica para el mejoramiento de procesos utilizando el enfoque Harrington y la Norma ISO 9004. Revista Universidad EAFIT, 41(139), 46-56.

Robayo Acuña, P. (2016). La innovación como proceso y su gestión en la organización: una aplicación para el sector gráfico colombiano. Suma de negocios, 7(16), 125-140.

Serrano Gómez, L. y Ortiz Pimiento, N. R. (2012). Una revisión de los modelos de mejoramiento de procesos con enfoque en el rediseño. Estudios Gerenciales, 28(125), 13-22.

Tarapuez, E. y Lima, C. (2013). Creatividad empresarial. Bogotá: Ecoe.

\section{BIBLIOGRAFÍA}

Bizagi (2019). Presentation mode and Presentation actions. Recuperado de https://help. bizagi.com/process-modeler/en/index.html?presentation_actions.htm

Escobar, B. y Gonzáles, J. M. (2007). Reingeniería de procesos de negocio: análisis y discusión de factores críticos a través de un estudio de caso. Revista Europea de Dirección y Economía de la Empresa, 16(3), 93-114. Recuperado de https://dialnet. unirioja.es/

Fernández Sánchez, E. (2005). Estrategia de innovación. Revista de investigación en gestión de la innovación y tecnología, 16, 141-142. Recuperado de https://dialnet.unirioja. es/servlet/articulo?codigo $=7054350$

García Gómez, M. C. y Portocarrero, D. C. (2019). Metodologías y herramientas de calidad para el mejoramiento continuo de las organizaciones. Universidad Santiago de Cali, 1-13. Recuperado de https://repository.usc.edu.co/bitstream /20.500.12421/1322/1/METODOLOG\%C3\%8DAS\%20Y\%20HERRAMIENTAS.pdf

Gavín, A. (2013). Un caso clásico de innovación: el carro de la compra de IDEO. El Miracielos. Recuperado de https://www.elmiracielos.com/innovacion/un-caso-clasico-deinnovacion-el-carro-de-la-compra-de-ideo/

Gestión (2012). Es tiempo de cuestionar la estrategia de la mejora continua. Gestión (21 de mayo). Recuperado de https://gestion.pe/impresa/cuestionar-estrategiamejora-continua-11324-noticia/

Gómez Fraile, F.; Vilar Barrio, J. F. y Tejero Monzón, M. (2002). Seis Sigma [segunda edición]. Madrid: Fundación Confemetal Editorial.

Kanchev, A. (2018). Improving the Internal Auditing Procedure by Using SIPOC Diagrams. Journal of Innovations and Sustainability, 4(2), 35-43. Recuperado de https://www. researchgate.net/publication/326539623_Improving_the_Internal_Auditing_ Procedure_by_Using_SIPOC_Diagrams 
Kleinsmann, M.; Valkenburg, R. y Sluijs, J. (2017). Capturing the Value of Design Thinking in Different Innovation Practices. International Journal of Design, 11(2), 25-40. Recuperado de http://www.ijdesign.org/index.php/IJDesign/article/view/2771

Lahajnar, S. y Rozanec, A. (2016). The Evaluation Framework for Business Process Management Methodologies. Journal of Contemporary Management Issues, 21(1), 47-69. Recuperado de https://moj.efst.hr/management/Vol21No1-2016/3-Laha jnar_Rozanec.pdf

Macías Rodríguez, M. (2017). El camino para innovar: cómo pasar de la idea al modelo de negocio creando valor para tus clientes. Barcelona: Deusto.

Medina León, A.; Nogueira Rivera, D.; Hernández Nariño, A. y Comas Rodríguez, R. (2019). Procedimiento para la gestión por procesos: métodos y herramientas de apoyo. Ingeniare, 27(2), 328-342. Recuperado de https://scielo.conicyt.cl/scielo. php?script=sci_arttext\&pid=S0718-33052019000200328

Nahabedian, L. (2017). Dynamic Update of Business Process Management. IEEE. International Conference on Software Engineering Companion (ICSE-C). Buenos Aires: IEEE.

Pérez Fernández de Velasco, J. A. (2012). Gestión por procesos [quinta edición]. Madrid: Esic.

Qualiex, Blog de la Calidad (13 de junio de 2018). MASP (Método de análisis y solución de problemas)-parte 1. Recuperado de https://blogdelacalidad.com/

Razzouk, R. y Shute, V. (2012). What is Design Thinking and Why Is It Important? Review of Educational Research, 82(3), 330-348. doi: 10.3102/0034654312457429

Rincón García, N.; Aguirre Mayorga, H. S. y Caballero Villalobos J. P. (2014). Business Process Management y Seis Sigma en el análisis de procesos: caso de estudio. Revista Venezolana de Gerencia (RVG), 19(67) 447-498. Recuperado de https:// www.redalyc.org/articulo.oa?id=29031856005

Sejzer, R. (2018). SIPOC: Mapeo de Procesos de Alto Nivel. Ronín Consultoría. Recuperado de https://qualityway.wordpress.com/2018/08/23/sipoc-mapeo-de-procesos-dealto-nivel-por-raul-sejzer/

Serrano, L. y Ortiz Pimiento, N. R. (2012). Una revisión de los modelos de mejoramiento de procesos con enfoque en el rediseño. Estudios Gerenciales, 28(125), 13-22. Recuperado de https://www.redalyc.org/pdf/212/21226279002.pdf

Serrano Ortega, M. y Blázquez Ceballos, P. (2014). Design Thinking [segunda edición]. Madrid: Esic. 